\title{
Endolaparoscopic retromuscular repair of smaller midline ventral hernias-too much for too little?
}

\author{
A. Sharma ${ }^{1}$ \\ Received: 5 June 2020 / Accepted: 19 June 2020 / Published online: 24 June 2020 \\ ๑) Springer-Verlag France SAS, part of Springer Nature 2020
}

The evolution of the retromuscular space as a preferred location for hernia repair of the abdominal wall has been long and eventful. The retromuscular hernia repair was initially described by Rene Stoppa for repair of bilateral inguinal hernias. Stoppa technique involved placement of a large sheet of polyester mesh in the preperitoneal or retrofascial space allowing for the intraabdominal pressure to secure the mesh between the peritoneum and abdominal wall [1]. The Open Rives-Stoppa retromuscular repair was developed by Drs. Jean Rives and Rene Stoppa in 1960's and later popularized by Dr. George Wantz [2]. Rives applied these priniciples for Ventral hernia repairs by placing the mesh in a similar retromuscular plane but above the arcuate line (posterior to the rectus muscle and anterior to the posterior rectus sheath) [3]. The retromuscular, prefascial location of mesh and Stoppa concept of giant prosthetic reinforcement of visceral sac were the principles for repair of inguinal hernias with minimal access surgery in Totally Extraperitoneal (TEP) and Transabdominal Preperitoneal (TAPP) techniques [4].

The enhanced view totally extraperitoneal technique (eTEP) was introduced by Jorge Daes [5] to improve access, increase surgical field exposure and provide better ergonomics for large complex inguinal hernias. The eTEP access technique was used to gain access to the retromuscular (retrorectus) space and endolaparoscopic retromuscular hernia repair for ventral and incisional hernias was reported to be feasible and effective (Ventral eTEP) [6]. Several newer approaches with Minimal access techniques have been reported for ventral/incisional abdominal wall hernias. (Laparoscopic transabdominal retromuscular (Ventral TARM), laparoscopic retromuscular ventral hernia repair (RMVH),

A. Sharma

asharma736@yahoo.in

1 Director, Endohernia Surgery, Institute of Laparoscopic, Endoscopic and Bariatric Surgery, Max Super Speciality Hospital, 1-2 Press Enclave Road, Saket, New Delhi 110017 India total extraperitoneal preperitoneal/retromuscular (Ventral TEP), enhanced view total extraperitoneal preperitoneal/ retromuscular (Ventral eTEP), transhernial total extraperitoneal/ preperitoneal/ retromuscular Mini or Less-Open Sublay repair (MILOS) or endoscopic variant (EMILOS) [7]. The surgical access in these procedures maybe endoscopic, laparoscopic or transhernial and location of mesh is retromuscular. These endolaparoscopic retromuscular mesh repairs are an attractive alternative as mesh is positioned extraperitoneally and an inexpensive polypropylene mesh may be used without need for penetrating fixation. These would necessarily translate into significant cost savings for the procedure and less postoperative pain to the patient.

The Open Rives Stoppa and Ventral eTEP repairs are alike as there is retromuscular mesh placement for hernia repair in both procedures. However, both procedures are different in terms of surgical technique. In the Open Rives Stoppa repair, surgical disruption of the midline (linea alba) has already occurred while making the midline incision for surgical access. In Ventral eTEP (eRives Stoppa) repair surgical access is from lateral ports and then there is deliberate and planned iatrogenic surgical division of bilateral posterior rectus sheaths (PRS) from xiphisternum to arcuate line to create the retromuscular plane for the mesh repair. In effect, with the endolaparoscopic approach the entire abdominal wall midline (PRS) needs to be divided only for the purpose of performing retromuscular mesh hernia repair.

It is evident that there is a price to be paid for the several advantages with an endolaparoscopic retromuscular repair. Primarily, the price is the significant collateral damage from iatrogenic division of the entire midline from xiphisternum upto arcuate line to prepare the landing zone for placement of the retrorectus mesh. Even for smaller hernias (upto $5 \mathrm{~cm}$ ) in endolaparoscopic retromuscular repairs, the midline cross over entails bilateral division of "normal" posterior rectus sheaths medially, from xiphisternum to arcuate line (about $25 \mathrm{~cm})$. 
The abdominal wall is anatomically configured to provide precise and optimal function from a myoaponeurotic system in which fascia, muscles, aponeuroses and sheaths operate synergistically [8]. The anterior and posterior rectus sheaths are derived from the bilaminar aponeuroses of the flat muscles of the anterolateral abdominal wall. The median intersection comprises the fibrous corpus termed the linea alba. In fact, the linea alba constitutes a complex system of decussating fibers derived from the bilaminar aponeuroses that cross the midline and interdigitate with contralateral fibers $[9,10]$. The abdominal wall is designed as a unitary functional system for protection of viscera, posture stabilization and movements of the trunk. A deliberate iatrogenic division of a component of this complex myoapeneurotic system will necessarily lead to loss of integrity and function. In endolaparoscopic retromuscular approaches, subsequent repair of the divided posterior rectus sheath with suture and inert mesh is a poor substitute to native functionally designed collagen tissue in vivo.

Endolaparoscopic retromuscular repair of ventral/incisional hernias is added armamentarium for the hernia surgeon. It is a novel approach that provides a sublay (posterior) mesh repair that is the preferred choice of many surgeons [11]. The eTEP technique provides access to the retrorectus(retromuscular) space in the medial compartment of the anterior abdominal wall. The retrorectus space provides a convenient and reproducible pathway to access the retromuscular extraperitoneal space for hernia repair at most locations in the anterior and posterolateral abdominal wall.

The endolaparoscopic retromuscular repairs have several advantages but also entail significant collateral damage to the midline of the abdominal wall. Therefore, it may be prudent to rationalize indications for this novel approach to hernia repair. Apart from several other indications, the endolaparoscopic retromuscular repair has been advocated as an option for surgical repair of small to moderate sized midline abdominal wall hernias too [7]. The ideal indication might be an existing significant compromise of the structural integrity and function of the abdominal midline. This maybe in the nature of a large midline hernia, a complex abdominal wall hernia requiring posterior component separation and symptomatic divarication of rectus abdominis muscle with a midline hernia. The endolaparoscopic retromuscular repair may also be a viable option to repair a recurrent abdominal wall hernia after previous anterior or intraperitoneal mesh repair. The endolaparoscopic retromuscular repair for small primary midline ventral wall hernias with an otherwise normal abdominal wall may be an overkill.
Funding No funding received.

\section{Compliance with ethical standards}

Conflict of interest The author declares no conflict of interest for the present work.

Ethical approval Ethics approval not applicable.

Human and animal rights Not applicable.

Informed consent Not applicable.

\section{References}

1. Stoppa R, Petit J, Abourachid H et al (1973) Original procedure of groin hernia repair: interposition without fixation of Dacron tulle prosthesis by subperitoneal median approach. Chirurgie 99(02):119-123

2. Wantz GE (1989) Giant prosthetic reinforcement of the visceral sac. Surg Gynecol Obstet 169(05):408-417

3. Rives J, Lardennois B, Pire JC (1973) Large incisional hernias. The importance of flail abdomen and of subsequent respiratory disorders. Chirurgie 99(08):547-563

4. Corbitt JD (1991) Laparoscopic herniorrhaphy. Surg Laparosc Endosc 1:23-25

5. Daes J (2012) The enhanced view-totally extraperitoneal technique for repair of inguinal hernia. Surg Endosc 26(4):1187-1189. https://doi.org/10.1007/s00464-011-1993-6

6. Belyansky I, Daes J, Radu VG et al (2018) A novel approach using the enhanced- view totally extraperitoneal (eTEP) technique for laparoscopic retromuscular hernia repair. Surg Endosc 32(3):1525-1532. https://doi.org/10.1007/s00464-017-5840-2

7. Bittner R, Bain K, Bansal VK et al (2019) Update of guidelines for laparoscopic treatment of ventral and incisional abdominal wall hernias International Endohernia Society IEHS Part B. Surg Endosc 33(10):3069-3139. https://doi.org/10.1007/s00464-01906907-7

8. Ruotolo F, Pinta ML, Gallinaro LS et al (2008) The anatomical structures of abdominal-wall continence. In: Crovella F, Bartone G, Fei L (eds) Incisional Hernia. Springer, Italia, pp 9-38

9. Askar OM (1977) Surgical anatomy of the aponeurotic expansions of the anterior abdominal wall. Ann R Coll Surg Engl 59:313

10. Rizk NN (1976) A new description of the anterior abdominal wall. Anat Rec 184:515

11. Holihan JL, Nguyen DH, Nguyen MT et al (2016) Mesh location in open ventral hernia repair: a systematic review and network meta- analysis. World J Surg 40(01):89-99

Publisher's Note Springer Nature remains neutral with regard to jurisdictional claims in published maps and institutional affiliations. 